\title{
AGENT-BASED DISTRIBUTED COLLABORATIVE MONITORING AND MAINTENANCE IN MANUFACTURING
}

\author{
Chun Wang ${ }^{1}$, Hamada Ghenniwa ${ }^{1}$, Weiming Shen ${ }^{1,2}$, Yue Zhang ${ }^{1}$ \\ ${ }^{\prime}$ Department of Electrical and Computer Engineering \\ The University of Western Ontario, London, Ontario, CANADA \\ ${ }^{2}$ Integrated Manufacturing Technologies Institute \\ National Research Council Canada, London, Ontario, CANADA \\ cwang28@engga.uwo.ca; hghenniwa@eng.uwo.ca; \\ weiming.shen@nrc.gc.ca; yzhan88@uwo.ca
}

\begin{abstract}
This paper proposes an agent-based approach for real-time collaborative monitoring and maintenance of equipments and machines. The proposed approach automates the maintenance management process by utilizing intelligent software agents. The maintenance schedules are achieved by automated real time negotiation among software agents representing geographically distributed field engineers and a broker. The paper presents a detailed system design and a prototype implementation of the collaborative monitoring and maintenance system which can be used by maintenance managers in manufacturing industry as a tool to improve the efficiency and quality of maintenance scheduling. Test results show that the proposed approach has potential for automating the maintenance management process in manufacturing environments.
\end{abstract}

\section{INTRODUCTION}

In today's modern manufacturing organization, the management of equipments and machines maintenance is one of the most important activities. Maintenance planning, scheduling and coordination focus on and deal with the preparatory tasks that lead to effective utilization and application of maintenance resources (Nyman, 2002). Traditionally this complex process is conducted by maintenance managers. After receiving reports of malfunctions or maintenance requirements, they allocate engineers for maintenance jobs at specific times and locations. Such allocation must take a number of factors into consideration, such as priority of and constraints between jobs, capability and availability of engineers, and costs. Although this manually controlled process is still widely adopted by manufacturers, it has a number of shortcomings in the context of today's global competitive manufacturing environments. Manufacturers are now under a tremendous pressure to improve their productivity and profitability. As an integrated part of production, maintenance management is required to be conducted in a more efficient way. In large scale or 
virtual enterprise manufacturing environments, plant floors are usually geographically distributed. In addition, environments in plant floors may change dynamically over time. Human maintenance managers may not be able to effectively handle this kind of large amount and dynamic changing information.

This paper proposes an agent-based approach for collaborative monitoring and maintenance of equipments and machines. The proposed approach automates the maintenance management process by utilizing intelligent software agents. The maintenance schedules are achieved by automated negotiation among software agents representing geographically distributed field engineers and a broker rather than a centralized scheduler.

The rest of this paper is organized as follows: Section 2 briefly discusses some background information regarding equipments and machines maintenance and agent based manufacturing; S ection 3 proposes a $\mathrm{n}$ a gent based collaborative monitoring and maintenance for equipments and machines; Section 4 describes the system architecture design and system components; Section 5 presents an implemented prototype environment; Section 6 provides a brief discussion and discusses the future work.

\section{RESEARCH BACKGROUND AND LITERATURE}

Effective maintenance of machines and equipments is very important for companies to sustain their manufacturing productivity and customer satisfaction. Traditional approaches, such as Total Productive Maintenance (TPM) (Venkatesh, 2003) and Reliability Centered Maintenance (RCM) (Moubray, 2001), mainly tackle this issue from the perspective of management science. TPM is a maintenance program concept evolved from Total Quality Management (TQM). Philosophically, it resembles Total Quality Management in several aspects. RCM is a highly structured framework that overturns many widely held beliefs about preventative maintenance. It was originally developed by the civil aviation industry and is now finding applications in various kinds of industrial and service organizations.

In light of the advances of Internet/Web technologies and e-business systems, eMaintenance has been considered as an integral part of e-Manufacturing. Koc and Lee proposed a system framework for the next generation e-maintenance systems, called Intelligent Maintenance System (IMS) (Koc and Lee, 2002). In this framework, the maintenance system is an Internet-based and Web-enabled predictive maintenance technology which consists of three levels. Firstly, at the product/machine/process level, the focus is on predictive intelligence. Secondly, at the system level the working equipments and machines are compared with symptoms under different conditions. Thirdly, at the enterprise level the focus is on the Web-enabled agent to achieve near-zero-downtime performance through smart asset optimization.

In today's highly distributed and dynamic manufacturing environments, agent based technologies have been proposed to overcome the limitations that traditional hierarchical and centralized control systems show. In this paradigm, the central controller is decomposed functionally or physically into several controllers, usually encapsulated as autonomous agents, each one devoted to a small portion of the overall system. The overall coordination is achieved by communication and negotiation among agents in the system. Once manufacturing systems are 
appropriately modeled as multi-agent systems and suitable negotiation protocols are adopted, such agent-based systems may yield a global performance which is flexible, robust, adaptive, and fault tolerant. A comprehensive survey of agent based manufacturing systems can be found in (Shen et al., 1999).

\section{COLLABORATIVE MAINTENANCE OF EQUIPMENTS AND MACHINES}

Agent based system technology has been proposed as a promising approach to address various issues in modern manufacturing industry, such as manufacturing scheduling and shop floor control (Shen et al., 2000; Shen, 2002), and supply chain management (Fox et al., 1993). In this paper we propose an agent based approach for collaborative maintenance equipments and machines with a focus on distributed, dynamic and open manufacturing environments.

In this approach, the maintenance management of equipments and machines is modeled as a multi-agent system. The system consists of five kinds of agents: diagnostic agent, broker agent, field engineer agent, directory facilitator agent, and help desk agent. Diagnostic agent is used to provide monitoring and diagnostic services to the equipments. It collects current failure and degradation data, analyzes the collect data, generates maintenance requirements and sends them to a broker agent. The broker agent plays a role similar to maintenance managers in the traditional maintenance process. After receiving the maintenance requirements, the broker agent checks with one of the directory facilitators to find capable field engineer agents currently registered within the system. The field engineer agent is a personal assistant of a field engineer. Information regarding the engineer's capabilities (types of services he can provide), availabilities (time table), and cost, is part of the knowledge of field engineer agent. A field engineer may dynamically change its profile and preferences. The broker agent negotiates with the field engineer agents to work out a suitable schedule for them to achieve the maintenance goals. In cases that the field engineers have technical difficulties during the process of equipment maintenance, they may locate a suitable remote help desk service by utilizing the negotiation mechanisms built in field engineer agents. The help desk services are provided by in-house engineers. Field engineers can communicate with in-house engineers through digital data, audio, and images which are encapsulated in a collaborative discussion mechanism.

At the system level, all agents need to register with one of the directory facilitator agents regarding their services provided. Director facilitator agents register with each other. In this way, any agent in the system can virtually find an agent $w$ ith the services it needs as long as it is alive in the system scope. In this approach, the equipment maintenance management is conducted as an automated procedure. Maintenance task planning, scheduling, and coordination are achieved by automated negotiation among agents. This mechanism provides an automation infrastructure for maintenance management process. At the operational level it can dramatically increase the process efficiency. In addition, the efficiency of automated negotiation mechanism makes use of real time distributed scheduling mechanisms, especially in large scale and dynamic environments. This will increase the quality of the maintenance schedules. 
The robust, flexible, and distributed natures of the agent based collaborative maintenance system make it easy to be deployed in various computer networks, even in global computing network environments. Although self-diagnostic agents can theoretically provide monitoring and diagnostic services to any equipment on the network, it is preferred that they sit close to the equipments or are connected directly to the equipments being monitored through a local network to provide reliable monitoring, diagnostic services with fast responses.

\section{SYSTEM ARCHITECTURE AND COMPONENTS}

\subsection{System Architecture}

In this work, we propose a six-layer system architecture (Figure 1) for the agent based distributed collaborative equipment maintenance system.

As shown in Figure 1, the Help Desk Services layer provides field engineers with remote help desk services. It contains help desk agents which can interact with field engineer agents to set up a conference session for in-house experts and a field engineer. The Maintenance Services layer provides maintenance services for the equipments. It contains field engineer agents representing a batch of field engineers and other necessary resources, such as tools and spare parts. Brokering Service layer consists of several agents which are responsible of finding suitable field engineers for a specific maintenance task and scheduling a time slot for it.

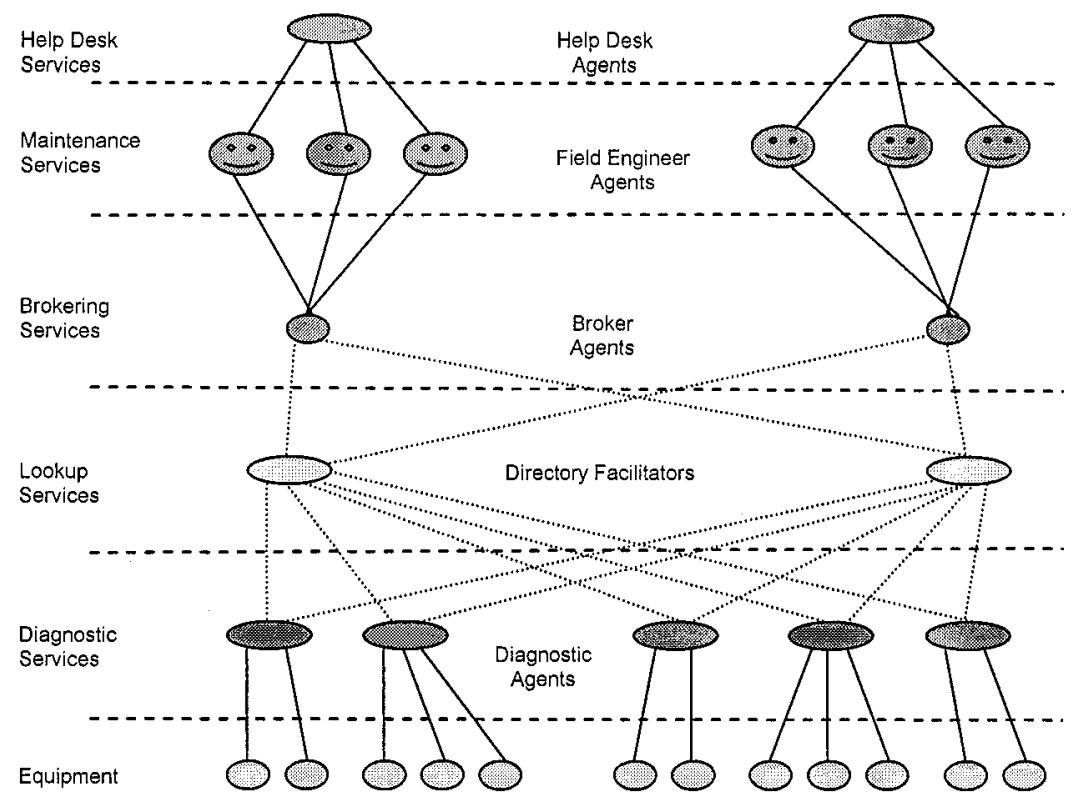

Figure 1- System structure for agent based collaborative maintenance

The Lookup Services layer contains Directory Facilitators agents. All agents in various service layers need to register with these Directory Facilitators agents. The Diagnostic Services layer includes diagnostic agents. They provide monitoring and 
diagnostic services to equipments. The Equipment layer consists of various equipments that need to be monitored and maintained.

\subsection{Agent Architecture}

The agent architecture adopted for the agents in our collaborative maintenance system is Coordinated, Intelligent Rational Agent (CIR-Agent) architecture (Ghenniwa, 2000). In the CIR-Agent model, an agent is an individual collection of primitive components. Each component is associated with a particular functionality that supports a specific agent's mental state as related to its goal. The agent's mental state regarding the reasoning about achieving a goal, in the CIR model, can be in one of the following:

1. Problem solving: determines the possible solutions for achieving a goal.

2. Pre-interaction: determines the number and the type of interdependencies as well as the next appropriate domain action.

3. Interaction: resolves the problems associated with the corresponding type of interdependencies. The mechanisms used in the interaction are called interactive devices.

4. Execution: affects the world.

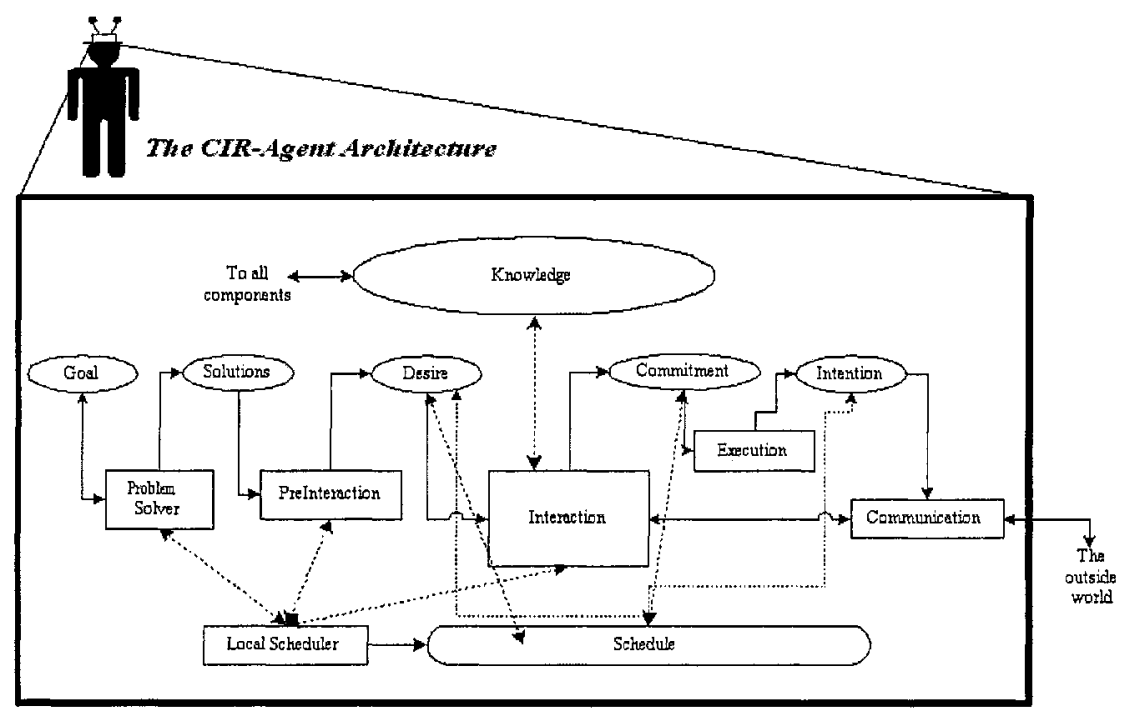

(a) Detailed Architecture of CIR-Agent

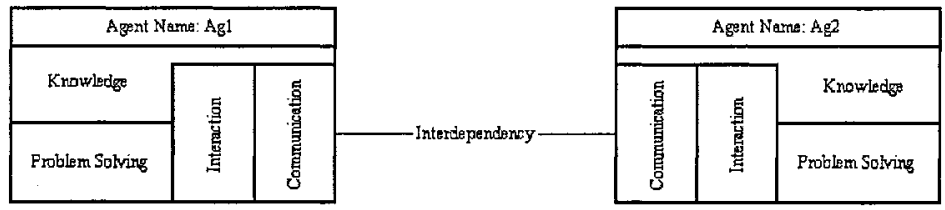

(b) Logical Architecture of CIR-Agent

Figure 2-Detailed and logical CIR-Agent architectures 
Based on these mental states, the CIR-Agent's architecture can be considered as a composition of four components: problem solver, pre-interaction, interaction, and execution. In the context of our agent based collaborative monitoring and maintenance system framework, we mainly focus on the problem solvers and interaction devices of different agents in the system.

\subsection{Diagnostic Agent}

The main functionalities of the diagnostic agent include monitoring, diagnostic and requirement generation services. In terms of CIR-Agent architecture, these should be encapsulated in the problem solver of diagnostic agents. In addition to monitoring current failures, diagnostic agents should monitor the degradation states of equipments as well. Based on the information, it generates different maintenance requirements with different priority in terms of the degrees of urgency.

The Interaction devices that diagnostic agents use to request maintenance services are FIPA Query protocols. Certainly, it needs to be connected to the equipments. The implementation of the connection between diagnostic agents and the equipments may depend on the data ports that the equipments can provide.

\subsection{Broker Agent}

Broker agents are used to schedule maintenance tasks received from diagnostic agents on capable field engineers. The problem solver of the broker agents is basically a scheduling engine. It assigns tasks to engineers by automated negotiation with field engineer agents. During the scheduling, several constraints, such as degree of urgency, field engineers' capability, availability, and costs, the availability of necessary tools need to be taken into consideration. At the same time, the scheduling engine selects the best possible solution that satisfies diagnostic agents' requirements according to their various objectives.

Broker a gents use FIPA Q uery p rotocols to r eceive maintenance requirements from diagnostic agents and FIPA Contract Net protocol to assign tasks to field engineers through field engineer agents.

\subsection{Field Engineer Agent}

Field Engineer agents are basically the personal assistants of field engineers. Field engineers may put their $p$ rofiles, preferences, $c$ urrent $s$ ituations, a nd a ny d ynamic changes into the field engineer agents. The problem solver of these agents is a scheduler dedicated to the field engineers. It is aware of all the user input information. It maintains an up-to-date schedule and historical data for a field engineer. It can automatically negotiate the task allocations with broker agents based on the user information it has. If they want, field engineers are able to involve into the negotiation process through a user interface. In addition, the problem solver of field engineer agents can locate a help desk agent and start a collaborative discussion with in-house engineers behind the help desk agent as well if a field engineer needs help during the maintenance process.

Interaction devices used by field engineer agents include FIPA Query protocols and FIPA Contract Net protocol. FIPA Contract Net protocol is used to n egotiate task allocation with broker agents. Both FIPA Query protocols and FIPA Contract Net protocol can be used to locate a help desk agent. If a field engineer agent knows which help desk agent it wants, it will go directly to the agent though FIPA Query protocols. Otherwise it will find a suitable one though FIPA Contract Net protocol. 


\subsection{Help Desk Agent}

The problem solver of help desk agents is a scheduler as well. It schedules a set of discussion sessions with field engineers based on the requirements from field engineer agents and their priority. In addition, it needs to consider the current availability and capability of in-house engineers. Help desk agents use FIPA Query protocols and FIPA Contract Net protocol to interact with field engineer agents. Real time discussion and collaborative design are also supported by help desk agent.

\subsection{Directory Facilitator agent}

Directory facilitator agents provide agent registration and look up service. They use standard FIPA Directory Facilitator interfaces to provide services.

\subsection{Collaboration between agents}

In this system a rchitecture, the collaboration mechanisms adopted between agents are economically inspired negotiation protocols, e.g. FIPA Contract Net and FIPA Query. Agents exchange information in the framework of these protocols. The preference models and negotiation strategies inside agents are not modeled in this system architecture. We leave them to domain specific implementations.

\section{PROTOTYPE IMPLEMENTATION}

To validate the proposed approach, a prototype environment has been implemented in a wireless network of mobile robots, desktop PCs, Pocket PCs, and a Smart Board. Figure 3 shows the actual prototype environment.

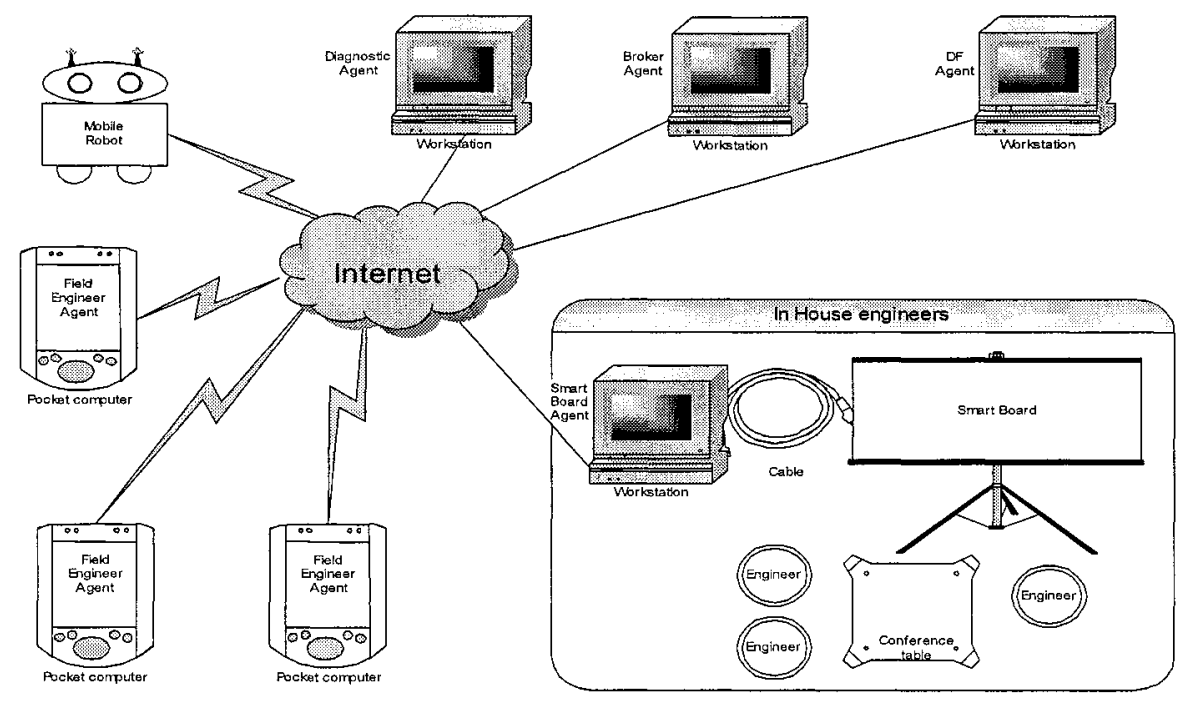

Figure 3-Agent based collaborative maintenance system prototype implementation In Figure 3, the equipment is a Magellan mobile robot which is connected to the diagnostic agent through a wireless network. iPAQ pocket $\mathrm{PCs}$ are used as the platforms of field engineer agents. The major collaborative discussion tool in this 
implementation is a smart board connected to a help desk agent. In this implementation we call the help desk agent as smart board agent, as it mainly provides the smart board service through which field engineers and in-house engineers discuss the maintenance tasks.

The diagnostic agent, the broker agent, the directory facilitator agent and the smart board agent are running on Pentium 4 PCs connected to the network.

Table 1 shows the hardware and software implementation details of the prototype environment.

Table 1-Hardware and software implementation details of the prototype

\begin{tabular}{|l|l|l|l|l|}
\hline Subsystem & $\begin{array}{l}\text { HardWare } \\
\text { Platform }\end{array}$ & $\begin{array}{l}\text { SoftWare } \\
\text { Platform }\end{array}$ & $\begin{array}{l}\text { Application } \\
\text { Package }\end{array}$ & $\begin{array}{l}\text { NetWork } \\
\text { Interface }\end{array}$ \\
\hline $\begin{array}{l}\text { Mobile } \\
\text { Robot }\end{array}$ & $\begin{array}{l}\text { Magellan } \\
\text { Robot }\end{array}$ & $\begin{array}{l}\text { Linux OS, } \\
\text { OmniORB }\end{array}$ & $\begin{array}{l}\text { Diagnostic } \\
\text { Information } \\
\text { Collection }\end{array}$ & Wireless LAN \\
\hline $\begin{array}{l}\text { Diagnostic } \\
\text { Agent }\end{array}$ & $\begin{array}{l}\text { Pentium 4 } \\
\text { PC }\end{array}$ & $\begin{array}{l}\text { Windows 2000, } \\
\text { JadeLeap }\end{array}$ & $\begin{array}{l}\text { Diagnostic } \\
\text { Agent Package }\end{array}$ & $\begin{array}{l}\text { Ethernet } \\
\text { Ethe }\end{array}$ \\
\hline $\begin{array}{l}\text { Broker } \\
\text { Agent }\end{array}$ & $\begin{array}{l}\text { Pentium 4 } \\
\text { PC }\end{array}$ & $\begin{array}{l}\text { Windows 2000, } \\
\text { JadeLeap }\end{array}$ & $\begin{array}{l}\text { Broker Agent } \\
\text { Package }\end{array}$ & $\begin{array}{l}100 \mathrm{M} \\
\text { Ethernet }\end{array}$ \\
\hline $\begin{array}{l}\text { Fngineer } \\
\text { Agent }\end{array}$ & PCAQ Pocket & $\begin{array}{l}\text { Windows CE, } \\
\text { JadeLeap }\end{array}$ & $\begin{array}{l}\text { Field Engineer } \\
\text { Agent Package }\end{array}$ & Wireless LAN \\
\hline $\begin{array}{l}\text { Smart Board } \\
\text { Agent }\end{array}$ & Pentium 4 & $\begin{array}{l}\text { Windows 2000, } \\
\text { JadeLeap }\end{array}$ & $\begin{array}{l}\text { Smart Board } \\
\text { Agent Package }\end{array}$ & $\begin{array}{l}100 \mathrm{M} \\
\text { Ethernet }\end{array}$ \\
\hline Smart Board & & & Serial Port \\
\hline DF Agent & Pentium 4 & $\begin{array}{l}\text { Windows 2000, } \\
\text { JadeLeap }\end{array}$ & JADE DF Agent & $\begin{array}{l}100 \mathrm{M} \\
\text { Ethernet }\end{array}$ \\
\hline
\end{tabular}

Figure 4 shows the graphical user interface of the broker agent. Four windows appear simultaneously in the interface for displaying Maintenance requirements, available field engineers, system status and the current schedule. The current schedule is presented in the form of a Gantt chart. The Gantt chart is the usual horizontal bar chart with the $\mathrm{x}$-axis representing the time and the $\mathrm{y}$-axis, the various field engineers (machines in terms of classical scheduling). The bars inside the Gantt chart represent operations of maintenance jobs. The operations belong to the same job have identical color. The coordinates of operations determined by identities of field engineers and times reflect the allocation of the operations to field engineers over time. 


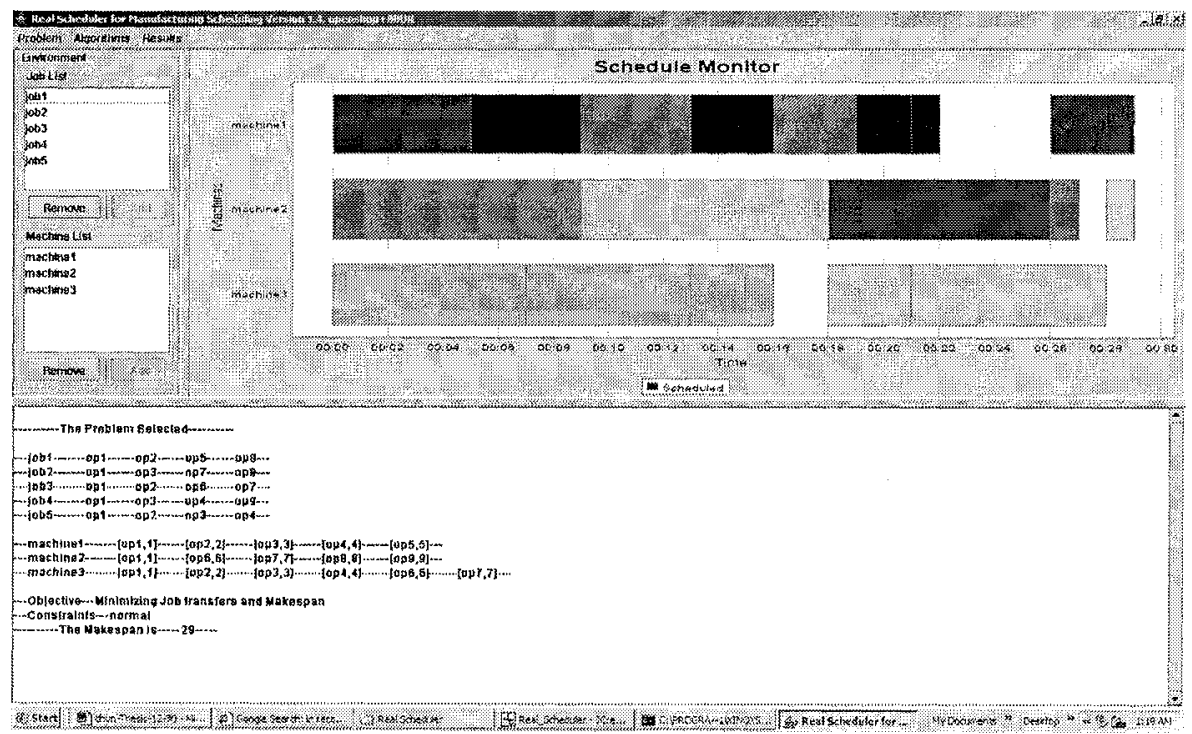

Figure 4 -User interface of broker agents

\section{DISCUSSION AND FUTURE WORK}

In this $\mathrm{p}$ aper, we p roposed, designed, a nd i mplemented a $\mathrm{n}$ a gent $\mathrm{b}$ ased sy stem to support distributed collaborative equipment monitoring and maintenance. The process is fully automated by utilizing intelligent agents. The system also supports the collaboration between field engineers and in-house engineers. Based on this agent based framework, efficient maintenance schedule can be generated by utilizing sophisticated distributed scheduling mechanisms to allocate maintenance tasks to field engineers over time. In addition, this highly open and flexible system framework can be easily integrated into e-Manufacturing and e-Business environments to provide maintenance services as part of the entire e-Manufacturing or e-Business framework.

The implemented system prototype environment has been tested in several scenarios of the maintenance management process. Results show that the proposed approach has potentials in terms of automating the maintenance management process in manufacturing environments.

For future extension, other negotiation mechanisms, such as various auctions, will be integrated into the system to provide further adaptability and efficiency in the dynamic, distributed environments. More effective distributed scheduling mechanisms, equipment monitoring and diagnostic techniques need to be integrated into the system as well. 


\section{ACKNOWLEDGEMENT}

We would like to acknowledge the financial support provided by Timelog International Inc., Barrie, Ontario, Canada (http://www.timeloginternational.com/) and Materials and Manufacturing Ontario (http://www.mmo.on.ca/). Also we would like to thank Wafa Ghoniam, Bashar Duheidel and Samer Hammoud who participated in the prototype implementation of the project.

\section{REFERENCES}

1. F ox, M .S., Chionglo, J.F., and B arbuceanu, M., The Integrated S upply C hain M anagement $S$ ystem Internal Report, Dept. of Industrial Engineering, University of ' Toronto, 1993, http://www.eil.utoronto.ca/public/iscm-intro.ps

2. Ghenniwa, H., and Kamel, M., Interaction Devices for Coordinating Cooperative Distributed System, Automation and Soft Computing, Vol.6, No.2, 2000, pp.173-184

3. Koc, M and Lee, J A S ystem F ramework for N ext-Generation E-Maintenance Systems, Intelligent Maintenance System Center, http://www.uwm.edu/Dept/IME/IMS, 2002.

4. Moubray, J. Reliability-Centered Maintenance, Industrial Process Inc. 2001.

5. Nyman, D., and Levitt, J., Maintenance Planning, Scheduling and Coordination, Industrial Process Inc. 2002.

6. Shen, W and Norrie, DH Agent-Based Systems for Intelligent Manufacturing: AState-of-the-Art Survey. Knowledge and Information Systems, an International Journal, 1(2), 129-156, 1999.

7. Shen, W., Lang, S., Korba, L., Wang, L., and Wong, B., Reference architecture for internet-based intelligent shop floor, Proceedings of the SPIE International Conference on Network Intelligence: Internet Based Manufacturing, Boston, MA, Nov. 8, 2000. Vol. 4208, PP. 63-71.

8. Shen, W., "Distributed Manufacturing Scheduling Using Intelligent Agents", IEEE Intelligent Systems, Jan./Feb., 2002, pp. 88-94

9. Smith, R.G. "The Contract Net Protocol: High-Level Communication and Control in a Distributed Problem Solver," IEEE Transactions on Computers, C-29(12):1104-1113, 1980

10.Venkatesh, J. An Introduction to Total Productive Maintenance, http:/www.plantmaintenance.com/articles/tpm_intro.shtml, 2003. 